\title{
Magnetic Confinement and Coupling in Narrow-Diameter Au-Ni Nanowires
}

\author{
Laura T. Schelhas ${ }^{1} \dagger$, Matthew J. Banholzer ${ }^{2} \dagger$, Chad A. Mirkin ${ }^{2 *}$, Sarah H. \\ Tolbert ${ }^{1,3,4 *}$
}

1. University of California-Los Angeles, Department of Chemistry and Biochemistry, University of California at Los Angeles, Los Angeles, CA 90095-1569, USA

2. Northwestern University, Department of Chemistry and International Institute for Nanotechnology. 2145

Sheridan Rd, Evanston, IL, 60208-3113, USA

3. Department of Materials Science and Engineering, University of California, Los Angeles, Los Angeles California 90095-1569, USA

4. California NanoSystems Institute, University of California, Los Angeles, Los Angeles California 90095-1569, USA

*Corresponding authors: tolbert@ chem.ucla.edu, chadnano@northwestern.edu

$\dagger$ †.T. Schelhas and M.J. Banholzer contributed equally to this work.

Here we examine magnetic coupling in layered magnetic/nonmagnetic nanowires created using electrochemical deposition into nanoporous templates. By utilizing reproducible and tunable deposition methods, various aspect ratios and spacing between magnetic domains were created. Low aspect ratio disks were then coupled to high aspect ratio rods to control the mechanism for spin flip in the disk component of the system. The orthogonal relationship between the magnetic easy axis of the disk and rod geometries creates a multistate system with both in-plane and out-of-plane easy axes by balancing magnetic shape anisotropy with dipole coupling between the two layers.

KEYWORDS: Nano-Magnets, Anodic Alumina, AAO, Ni-Au Nanowires, Shape Anisotropy

\subsection{INTRODUCTION}

The ability to organize magnetic and nonmagnetic materials across different length scales has led to the synthesis of devices that show novel magnetic effects.[1,2] Magnetoresistive spin-valves are a common example, where spin transfer between layers of magnetic materials can result in changes in the electrical resistance of the material.[1,2] In spin-valve 
devices, resistance is dependent on the relative spin orientation of two separated magnetic layers. The first magnetic layer, the free layer, can switch between magnetic orientations, while the second layer, the pinned layer, contains spins that are oriented in a fixed direction. These two magnetic layers are separated by a nonmagnetic spacer layer.[3] Parallel spins in the two magnetic regions allow for low device resistance to an injected spin-polarized current, while antiparallel spins produce a high resistance; this effect is known as giant magnetoresistance (GMR).[2,4,5] Previous work has built on the basic geometry described above by utilizing a perpendicular spin polarizer.[6-10] The polarizer allows for a more energy efficient precessional out-of-plane switching of the magnetic spin, compared to the less favorable in-plane switching. Based on these ideas, there have been large scale demonstrations of lithographically produced spin-valve arrays with dimensions in the range of $100 \times 200 \mathrm{~nm}^{2} \cdot[7,11]$

Here we present a fundamental study of coupling in nanosized magnetic layers, motivated by the efforts in spinvalve devices. We specifically address the following question. As the size of the active elements is reduced to the single domain limit (here $\sim 50 \mathrm{~nm}$ ), can we control the mechanism and barrier for spin flip using coupled nanosized magnetic domains? To do this, we vary the spacer distance between a disk and a rod to study the effects on the coupling between the two magnetic components. The disks and rods are created by electrochemical deposition of magnetic and non-magnetic metals into anodic alumina pores. This method allows us to precisely control all dimensions of the magnetic domains for coupling measurements, making it an ideal system for a fundamental study of the coupling of various sample geometries. However, previous spin-torque measurements on electroplated multilayer systems that have shown effects are not large in these materials. Therefore, the disk/rod couples used here should be consider a geometric model system that is not likely to be useful for actual devices.[12-14]

\subsection{Materials and Methods}

Magnetic nanowires were synthesized in a similar manner to previously published work.[15-18] Circular sheets of AAO $(13 \mathrm{~mm})$ with $45 \mathrm{~nm}$ inner diameter pores were purchased from Synkera Inc. and used as templates for the rod synthesis (note that the labeled pore size on the AAO used here was $35 \mathrm{~nm}$, but significant variation is observed). Ag (200 $\mathrm{nm}$ ) was evaporated on one side of each template and used as a working electrode to deposit the rod materials. The templates were mounted into an electrochemical cell, and $2 \mathrm{C}$ of a Ag "buffer" layer was deposited under constant potential electrolysis at $-1150 \mathrm{mV}$ (Technic Inc. $1025 \mathrm{RTU}$ plating solution diluted to $80 \%$ concentration was used). The electrochemical cell was thoroughly rinsed, and Au and Ni sections were alternately plated (at -970 and -910 mV, employing Technic Inc Orotemp 24 Rack and Nickel Sulfamate RTU solutions, respectively) until the desired nanowire segment lengths were achieved. The Ag backing/buffer layer was then etched in a 3:1:1 mixture of $95 \%$ ethanol to $30 \% \mathrm{H}_{2} \mathrm{O}_{2}\left(\right.$ in $\mathrm{H}_{2} \mathrm{O}$ ) to $28 \% \mathrm{NH}_{4} \mathrm{OH}$ (in $\mathrm{H}_{2} \mathrm{O}$ ). The 
alumina network was further etched off a small portion of each sample and the freed nanowires were examined in a Hitachi 4800 SEM (Fig. 1). The remainder of the wires remained within the alumina membrane for magnetic characterization. M-H curves were measured by SQUID Magnetometry using a Quantum Design MPMS with DC detection. Samples were measured with the field applied parallel and perpendicular to the pore long axis. A TJA Radial Iris 1000 ICP-OES was used to determine the relative concentrations of $\mathrm{Ni}$ in the coupled wires. This allowed us to subtract out the magnetic contribution of the rods in coupled rod-disk systems. Samples for ICP-OES were digested in $300 \mu \mathrm{l}$ of Optima $\mathrm{HCl}$ and $100 \mu \mathrm{l}$ of Optima $\mathrm{HNO}_{3}$ and diluted with $8 \mathrm{ml}$ of $5 \%$ Optima $\mathrm{HNO}_{3}$ after 3 hours heating at $85^{\circ} \mathrm{C}$.

\subsection{Results and Discussion}

The shape of magnetic materials plays an important role in determining the overall magnetic properties. This is most prominently manifested in nanosystems as these are frequently single domain magnets. Typically, the long axis of a nanostructure provides the more energetically favorable orientation for spin alignment; this is commonly named the easy magnetic axis. The origin of this effect occurs when magnetic charges or poles at the surface of the material create a magnetic field that acts to oppose the internal magnetization of the sample.[1] This demagnetizing field is dependent on the shape of a nanostructure; as the aspect ratio increases, this demagnetization factor along the long axis approaches zero. Since the demagnetization factor is inversely proportional to ease of magnetization, it becomes easier to magnetize the sample in the long direction, thus creating the easy axis. Note that this shape anisotropy is distinct from magnetocrystalline anisotropy, which arises from bond anisotropy within the crystal lattice.[1] While both are present in magnetic nanosystems, shape anisotropy generally dominates over magnetocrystalline anisotropy in highly non-spherical nanosized objects.[1] In our system, we deliberately synthesized rods and disks (aspect ratio $>3: 1$ and $<1: 2$, respectively) that should exhibit complementary easy axes: along the long axis of the rod structure or in the plane of the disk. 

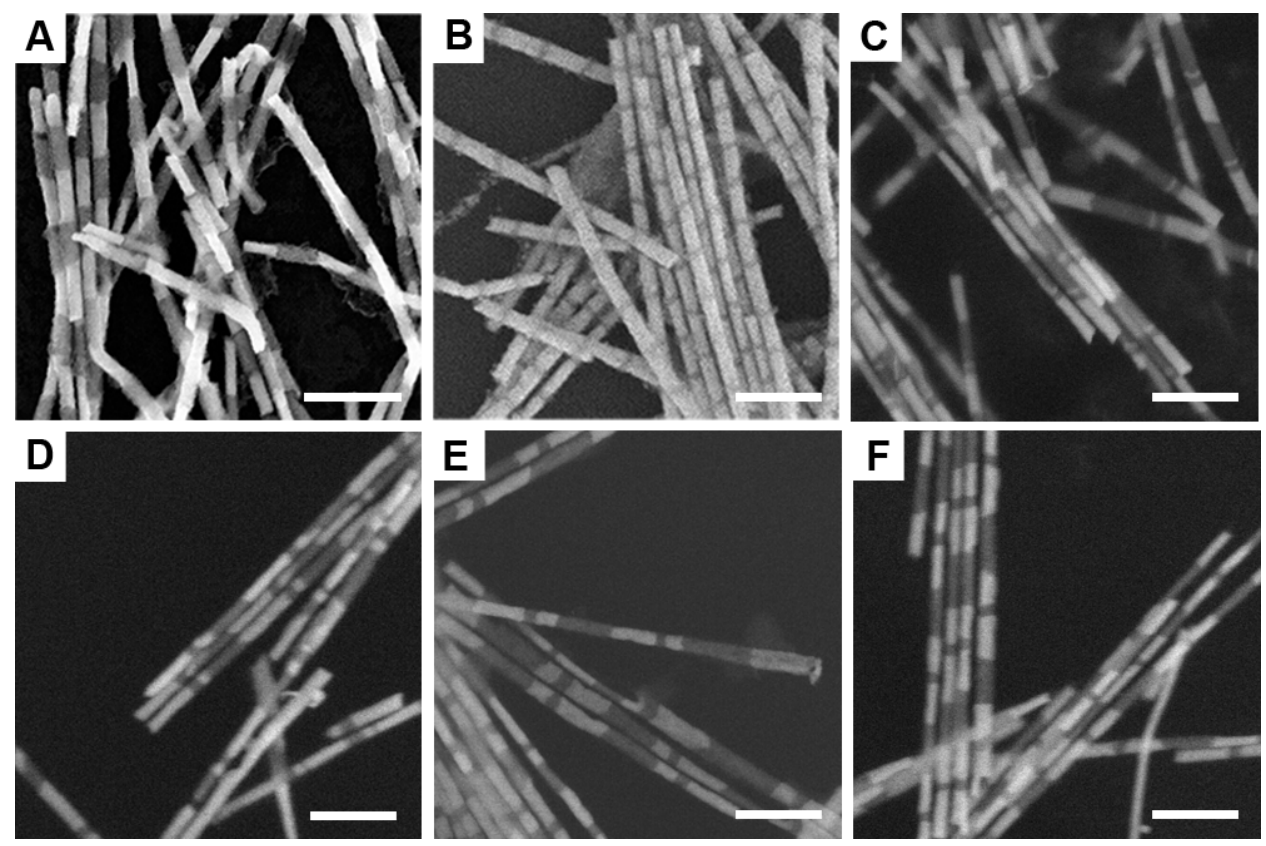

Figure 1. Selected SEM images of nanorods used in study. (A) $153 \mathrm{~nm}$ long Ni rods separated by $155 \mathrm{~nm}$ Au segments, diameter $=48 \mathrm{~nm}$ (B) $19 \mathrm{~nm}$ long Ni disks separated by $143 \mathrm{~nm}$ Au segments, diameter $=45 \mathrm{~nm}(\mathrm{C})$ Pairs of $148 \mathrm{~nm}$ long Ni segments and $26 \mathrm{~nm}$ long Ni segments separated by $11 \mathrm{~nm} \mathrm{Au} \mathrm{segments.} \mathrm{(D)} \mathrm{Pairs} \mathrm{of} 161 \mathrm{~nm}$ long Ni segments and $30 \mathrm{~nm}$ long Ni segments separated by 29 nm Au segments. (E) Pairs of 168 nm long Ni segments and 32 nm long Ni segments separated by $52 \mathrm{~nm}$ Au segments. (F) Pairs of $168 \mathrm{~nm}$ long Ni segments and $31 \mathrm{~nm}$ long Ni segments separated by $124 \mathrm{~nm}$ $\mathrm{Au}$ segments. In (C-F), each Ni-rod Au-spacer Ni-disk pair is separated by approximately $140 \mathrm{~nm}$ of Au. Scale bars are 300 $\mathrm{nm}$ in all cases.

Nanostructures of varying aspect ratios were synthesized with the goal of finding structures that showed the highest magnetic anisotropy (i.e. high remanence and coercivity in only one axis) (Fig. 1). The easy magnetic axis of the high aspect ratio rods and low aspect ratio disks were identified by examining hysteresis in $\mathrm{M}-\mathrm{H}$ curves. An example of a hysteresis loop for an optimized rod sample is shown in figure $2 \mathrm{~A}$. These data indicate a single magnetic easy axis along the long axis of the rod, as shown by a high remanence (0.936) and coercivity (1660 Oe) when the magnetic field is applied parallel to the long axis of the rod. Conversely when the magnetic field is applied perpendicular to the long axis of the rod, both remanence (0.071) and coercivity (240 Oe) are low. Alternatively, disk segments exhibit a planar distribution of easy axes within the plane of the disk (Fig 2B). This is evidenced by a larger remanence when the magnetic field is applied parallel to the plane of the disk (0.541) rather than when applied perpendicular to the plane of the disk (0.293). While the anisotropy is much weaker in disk segments compared to the rod structures, this is expected because disks contain a plan distribution of easy 
axes, rather than a single easy axis. The result is also consistent with previous findings concerning magnetic shape anisotropy of similar structures.[19-22]

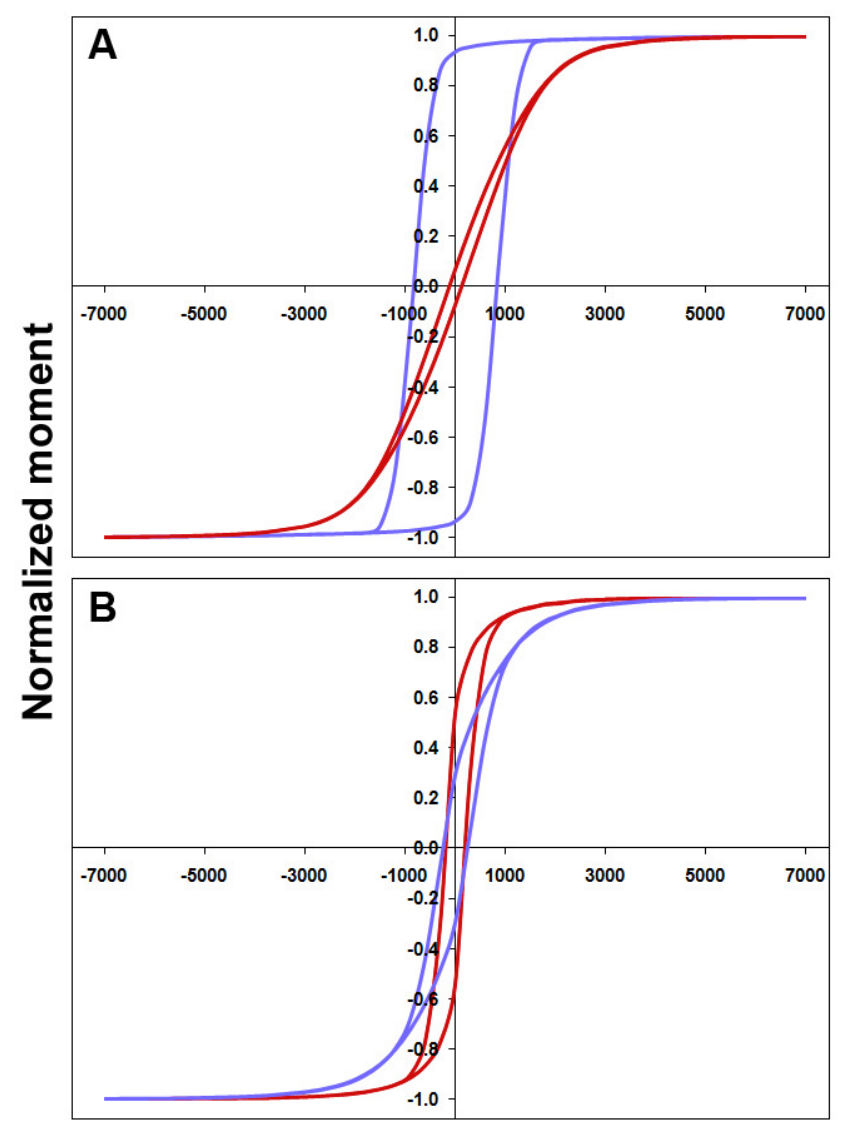

Field (Oe)

Parallel orientation

Perpendicular orientation

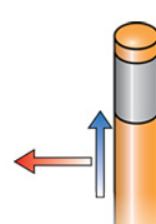

Figure 2. Magnetic response curves obtained on $153 \mathrm{~nm}$ long Ni rods and $19 \mathrm{~nm}$ thick Ni disks, both created in pores $45 \mathrm{~nm}$ in diameter. (A) High aspect ratio rods aligned parallel to the applied magnetic field exhibit a coercivity of 1660 Oe and a remanence of 0.936 . Under perpendicular magnetization these rods exhibit a coercivity of only 240 Oe and a remanence of 0.071. (B) Low aspect ratio disks with the plane of the disk orientation parallel to the applied magnetic field (and thus the pore axis perpendicular to the field) exhibit coercivity of 390 Oe and remanence of 0.541 . When the applied filed is parallel to the pore axis (perpendicular to the plane of the disk), the coercivity is 480 Oe and the remanence is only 0.293 . This implies an easy axis that lies in the plane of the disks. The bottom of the figure contains are cartoon illustrating parallel and perpendicular magnetic fields. 
Table I. Effects of aspect ratio on magnetic anisotropy

\begin{tabular}{llll} 
Rods & Aspect Ratio & Length $(\mathrm{nm})$ & $\mathrm{M}_{\mathrm{r}}$ length $/ \mathrm{M}_{\mathrm{r}}$ diameter \\
\hline R1 & 2.0 & 70 & 3.7 \\
$\mathrm{R} 2$ & 3.2 & 153 & 13.4 \\
$\mathrm{R} 3$ & 4.0 & 1330 & 0.8 \\
& & & \\
& & & \\
Disks & Aspect Ratio & Length $(\mathrm{nm})$ & $\mathrm{M}_{\mathrm{r}}$ diameter $/ \mathrm{M}_{\mathrm{r}}$ thickness \\
\hline & & & \\
D1 & 0.2 & 8 & 0.8 \\
D2 & 0.4 & 19 & 1.9
\end{tabular}

In determining these optimized aspect ratios, a range of disk and rod samples were examined. Table I summarizes the magnetic anisotropy measured for 5 particularly illustrative samples, 3 rods and 2 disks. The table shows aspect ratio, absolute length or thickness, and the ratio of remanent magnetization along the easy magnetic axis compared to the remanence in the hard direction. For rod-like structures, this is a ratio of the remanence parallel to the long axis of the rod divided by the remanence perpendicular to the rod. For disks, the opposite ratio is used - the table reports the remanence parallel to the plane of the disk divided by the remanence perpendicular to the plane of the disk. Defined in this way, the highest ratio always indicates the most ideal structure. For rod like structures, an aspect ratio of 2:1 produces modest anisotropy, but the anisotropy increases significantly when the aspect ratio increases to $3: 1$ or higher. Samples similar to R2 were thus used as the rod-like segment in all work on coupled structures. When the dimensions get to be too large - either too long or too wide and too long, as seen in R3 - the remanent anisotropy plummets because the rods no longer contain a single magnetic domain and shape anisotropy no longer dominates the domain alignment. For disks, we expected that thinner structures would be better, but comparison of samples D1 and D2 indicates that the disks can be too thin. This likely stems from roughness within the nanowire stack. While our method can be used to precisely plate a desired thickness of metal, if the nucleating surface is not flat, the resulting disk-like structure will not be perfectly flat. Shapes that are thick compared to the roughness thus show higher magnetic anisotropy. Samples similar to D2 were thus used as the disk-like segment in all work on coupled structures.

As previously stated, our goal in this work is to examine how a disk/rod couple can be used to tune the static barrier for spin flip in a nanosized element that could serve as a model for a free layer in a spin-valve type structure. By coupling the disk's horizontal easy axis with the rod's vertical easy axis it should be possible to create a range of intermediate spin state, 
potentially leading to a magnetic system with both in-plane and out-of-plane easy axes. (Fig. 3A). We note that in this work, the cross-section of our stack is circular, so that there are no preferential in-plane alignment directions. In any real device using these ideas, an oval or rectangular cross-section would be needed to create just two in-plane easy-axes, coupled to one out-of-plane easy axis. The goal is to use the out-of-plane easy axis to control the mechanism for spin flip and to reduce the energetic barrier for magnetization reversal by balancing magnetic shape anisotropy with dipole coupling between layers. Coupling of the spins between the two segments pulls the free layer's magnetization out of the plane of the disk. The magnetization is transiently parallel to the high aspect ratio pinned layer's easy axis and allows the disk to more easily leave its initial magnetization and relax back down to a new magnetization direction. We note that because we are using stacks with a circular cross-section, the barrier for in-plane magnetization reversal is already much reduced compared to a more standard rectangular cross-sections, so any beneficial effects of this coupling should only be increased in real systems. Theory has predicted that the use of a perpendicularly aligned element creates a more energy efficient device[6-10]. This geometry has also been experimentally realized in larger lithographically prepared systems[7,11].

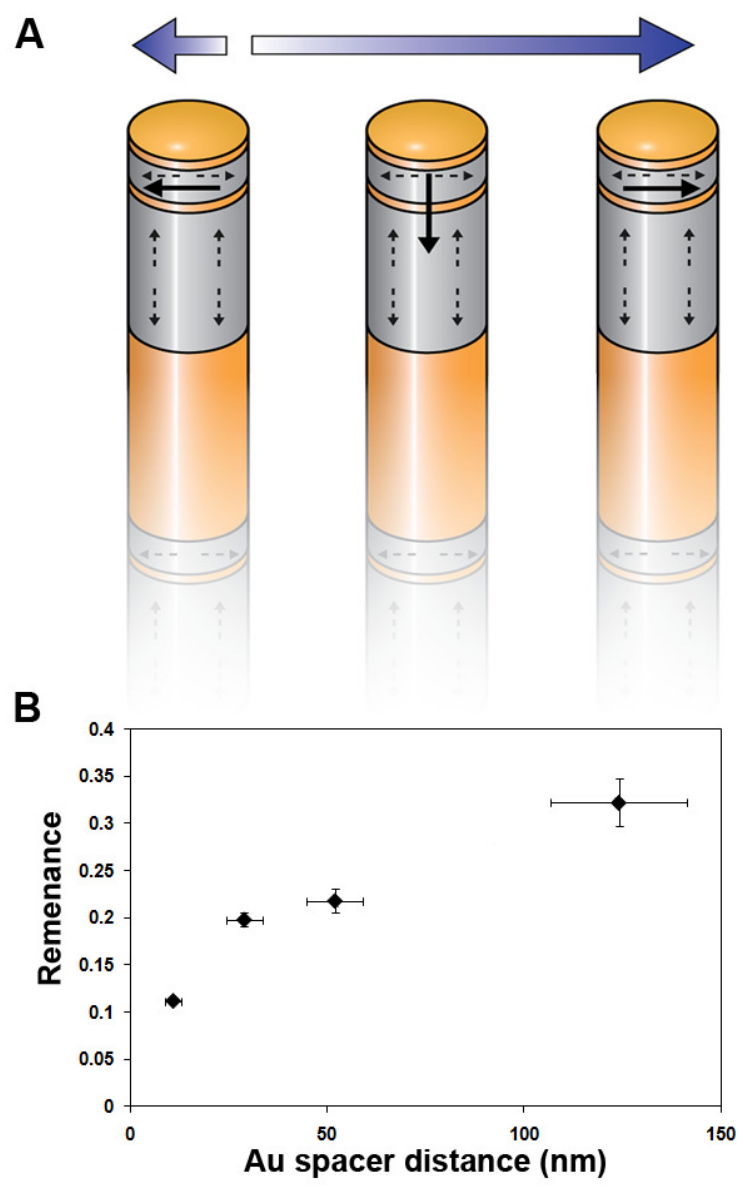


Figure 3. Coupling of high aspect ratio rods and low aspect ratio disks. (A). Schematic of coupling between disk and rod sections. The purple arrows indicate the magnetic field direction (perpendicular to the long axis of each nanowire), bold black arrows indicate magnetization of disks, dotted grey arrows indicate the easy axis of each magnetic segment. (B) Change in the remanant magnetization of the Ni disks with Au spacer distance. The data indicates that disk-rod coupling can be used to tune the propensity for spins to be aligned perpendicular to the plane of the disk.

We tested these ideas by varying the proximity of the disk element to the rod element through control of the spacer distance between the two magnetic components; static magnetization measurements were then used to probe the magnetic coupling. We began by fabricating nanostructures in which the high and low aspect segments are placed in close proximity to one another (Fig. 1 C-E). When the nanostructures are placed in a magnetic field perpendicular to the longwire axis (i.e. along the plane of the low aspect ratio disks), we observe that the remanent magnetization of disks decreases with decreasing spacing between the pinned rod layer and the free disk layer (Fig. 3B). This data was obtained by subtracting out the signal from the rod segments using magnetization data obtained on uncoupled rods with the same dimensions. Scaling of magnetization curves between pure rod and rod-disk samples was done based on the total $\mathrm{Ni}$ content in the rods of each sample. The procedure allows us to more directly see the effect of coupling on the low aspect ratio segments.

The result is a multi-state magnetic system where coupling to the easy axis of the high aspect ratio rod effectively creates an extra out-of-plane easy magnetic axis parallel to the longwise axis in addition to the in-plane easy axes created by the shape anisotropy of the disks. Since the intermediate spin state allows for lower energy switching, magnetic remanence is decreased.

\subsection{CONCLUSION}

In this work we have demonstrated how one can reproducibly and rationally design and synthesize narrow diameter $(<50 \mathrm{~nm})$ multilayer magnetic nanowires. Through controlled placement of magnetic and nonmagnetic sections, we have experimentally studied the coupling of various geometries of magnetic materials. We have shown that by coupling high aspect ratio magnetic rods with low aspect ratio magnetic disks we can increase the propensity of disk to switch out-of-plane by coupling to the rod. The results are complementary to previous work on spin systems utilizing dynamic perpendicular spin polarizers, in that those systems aim to control the precession of electrons moving through a spin-torque device. Here we aim to modify the static barriers for magnetization switch; presumably both methods could be used in combination in an optimized device. 


\section{ACKNOWLEDGMENTS}

The authors wish to thank Ignacio Martini for assistance with SQuID magnetometry. SHT acknowledges support from from the NSF under Cooperative Agreement Award EEC-1160504 and from the WIN center (funded by the UC Discovery program, Intel, and the NERC). . CAM acknowledges an NSSEFF Fellowship from the DoD and support from the NSF MRSEC program and the DOE EFRC program through the Northwestern University Non-equilibrium Research Center.

\section{REFERENCES}

[1] N.A. Spaldin, Magnetic Materials: Fundamentals and Device Applications, 1st ed., Cambridge University Press, New York, 2003.

[2] G.A. Prinz, Magnetoelectronics, Science (80-. ). 282 (1998) 1660-1663.

[3] G. Binasch, P. Grunberg, F. Saurenbach, W. Zinn, Enhanced Magnetoresistance in Layered Magnetic-Structures with Antiferromagnetic Interlayer Exchange, Phys. Rev. B. 39 (1989) 4828-4830.

[4] E.Y. Tsymbal, D.G. Pettifor, Perspectives of giant magnetoresistance, in: Solid State Physics, Vol 56, Elsevier Academic Press Inc, San Diego, 2001: pp. 113-237.

[5] M. Johnson, Spintronics, J. Phys. Chem. B. 109 (2005) 14278-14291.

[6] a. D. Kent, B. Özyilmaz, E. del Barco, Spin-transfer-induced precessional magnetization reversal, Appl. Phys. Lett. 84 (2004) 3897. doi:10.1063/1.1739271.

[7] C. Papusoi, B. Delaët, B. Rodmacq, D. Houssameddine, J.-P. Michel, U. Ebels, et al., 100 Ps Precessional SpinTransfer Switching of a Planar Magnetic Random Access Memory Cell With Perpendicular Spin Polarizer, Appl. Phys. Lett. 95 (2009) 072506. doi:10.1063/1.3206919.

[8] D. Houssameddine, U. Ebels, B. Delaët, B. Rodmacq, I. Firastrau, F. Ponthenier, et al., Spin-torque oscillator using a perpendicular polarizer and a planar free layer., Nat. Mater. 6 (2007) 441-7. doi:10.1038/nmat1905.

[9] K.J. Lee, O. Redon, B. Dieny, Analytical investigation of spin-transfer dynamics using a perpendicular-to-plane polarizer, Appl. Phys. Lett. 86 (2005) 022505. doi:10.1063/1.1852081.

[10] U. Ebels, D. Houssameddine, I. Firastrau, D. Gusakova, C. Thirion, B. Dieny, et al., Macrospin description of the perpendicular polarizer-planar free-layer spin-torque oscillator, Phys. Rev. B. 78 (2008) 024436. doi:10.1103/PhysRevB.78.024436.

[11] T. Seki, S. Mitani, K. Yakushiji, K. Takanashi, Magnetization reversal by spin-transfer torque in $90^{\circ}$ configuration with a perpendicular spin polarizer, Appl. Phys. Lett. 89 (2006) 172504. doi:10.1063/1.2369648.

[12] A. Blondel, J.P. Meier, B. Doudin, J. Ansermet, Giant magnetoresistance of nanowires of multilayers, Appl. Phys. Lett. 65 (1994) 3019-3021.

[13] L. Piraux, J.M. George, J.F. Despres, C. Leroy, E. Ferain, R. Legras, et al., Giant magnetoresistance in magnetic multilayered nanowires, Appl. Phys. Lett. 65 (1994) 2484. doi:10.1063/1.112672.

[14] K. Liu, K. Nagodawithana, P.C. Searson, C.L. Chien, Perpendicular giant magnetoresistance of multilayered Co/Cu nanowires, Phys. Rev. 51 (1995) 7381-7385. 
[15] C.R. Martin, Nanomaterials: A Membrane-Based Synthetic Approach, Science (80-. ). 266 (1994) $1961-1966$.

[16] D. Routkevitch, T. Bigioni, M. Moskovits, J.M. Xu, Electrochemical Fabrication of CdS Nanowire Arrays in Porous Anodic ALuminum Oxide, J. Phys. Chem. 100 (1996) 14037-14047.

[17] S.J. Hurst, E.K. Payne, L. Qin, C.A. Mirkin, Multisegmented One-Dimensional Nanorods Prepared by HardTemplate Synthetic Methods, Angew. Chem. Int. Ed. 45 (2006) 2672-2692.

[18] M.J. Banholzer, L. Qin, J.E. Millstone, K.D. Osberg, C.A. Mirkin, On-wire lithography: synthesis, encoding and biological applications, Nat. Protoc. 4 (2009) 838-848.

[19] M. Chen, L. Sun, J.E. Bonevich, D.H. Reich, C.L. Chien, P.C. Searson, Tuning the response of magnetic suspensions, Appl. Phys. Lett. 82 (2003) 3310-3312. doi:10.1063/1.1569429.

[20] H.-P. Liang, Guo, J.-S. Hu, C.-F. Zhu, L.-J. Wan, C.-L. Bai, Ni-Pt Multilayered Nanowire Arrays with Enhanced Coercivity and High Remanence Ratio, Inorg. Chem. 44 (2005) 3013-3015. doi:10.1021/ic0500917.

[21] M. Chen, P.C. Searson, C.L. Chien, Micromagnetic behavior of electrodeposited Ni/Cu multilayer nanowires, J. Appl. Phys. 93 (2003) 8253-8255.

[22] M. Chen, C.-L. Chien, P.C. Searson, Potential Modulated Multilayer Deposition of Multisegment Cu/Ni Nanowires with Tunable Magnetic Properties, Chem. Mater. 18 (2006) 1595-1601. 